\title{
VALORIZACIÓN DE RESIDUOS GENERADOS EN LA AGROINDUSTRIA EN GUANAJUATO APLICANDO PRINCIPIOS DE ECOLOGÍA INDUSTRIAL
}

\author{
Revaluation of wastes generated in agroindustry in Guanajuato applying principles of industrial ecology
}

\author{
Estephanie María de los Ángeles SOLÍS JIMÉNEZ1, \\ Gemma CERVANTES $^{2}$ y Sylvie TURPIN MARION ${ }^{1 *}$
}

${ }^{1}$ Universidad Autónoma Metropolitana-Unidad Azcapotzalco. Av. San Pablo 180, Col. Reynosa Tamaulipas, 02200 Ciudad de México, México

${ }^{2}$ Facultad de Agronomía, Universidad de la Salle Bajío, Campus Campestre, Av. Universidad 602, Col. Lomas del Campestre, 37150 León, Guanajuato, México

*Autora para correspondencia: stm@correo.azc.uam.mx

(Recibido enero 2017; aceptado diciembre 2018)

Palabras clave: industria porcina, simbiosis industrial, sinergias, aprovechamiento

\section{RESUMEN}

En el municipio de Pénjamo, Guanajuato, el sector productivo más importante es el agroindustrial, destacando la industria porcina. Sin embargo, ésta no cuenta con una gestión adecuada de residuos como pelo, pezuñas y párpados de cerdos, que son susceptibles de aprovechamiento. Este trabajo propone alternativas de valorización de estos residuos aplicando la ecología industrial, que busca establecer alternativas para el cierre de ciclo de materia y el aprovechamiento de energía en sistemas industriales. De esta manera construye sinergias entre empresas existentes (o bien entre una empresa existente y una nueva), y propicia el aprovechamiento de los flujos residuales de una empresa como materia premia por otra. En este caso, se analizó el entorno de un clúster agroindustrial de Pénjamo y se propusieron nuevas sinergias: la elaboración de aminoácidos con el pelo de cerdo, la fabricación de grenetina a partir de pezuñas y párpados, la transformación en biogás y/o energía eléctrica de purines de granjas porcinas y la obtención de láminas y tableros utilizando el bagazo de agave. Asimismo, se propusieron intercambios que podrían contribuir a la formación de otras empresas; por ejemplo, crear una planta de composta a partir de residuos orgánicos de invernaderos, mermas de semillas y alimentos. Con las sinergias propuestas, se pueden reducir los residuos generados por el sector agroindustrial y aprovechar residuos generados por empresas de giros diferentes al porcino, lo que conllevaría un beneficio ambiental y un beneficio económico.

Key words: swine industry, industrial symbiosis, synergies, reuse

\begin{abstract}
In the municipality of Pénjamo, Guanajuato, Mexico, the agroindustry is the most important productive sector, especially the swine industry. However, wastes form this industry still lack a proper management. There are residues such as hair, hooves and eyelids of pigs that could provide additional value and opportunities. This work proposes alternatives for the valorization of swine production residues, applying industrial
\end{abstract}


ecology, which seeks to establish alternatives for the closing of the cycle of matter and energy utilization in industrial systems, thus building synergies between existing companies (or between an existing company and new ones) and encouraging residual flows being produced by one and reutilized as raw material by another. In this case, the environment of an agro-industrial cluster in Pénjamo was analyzed and new synergies were proposed: the development of amino acids using pig hair, the manufacture of gelatine from hooves and eyelids, the transformation of slurry from porcine farms into biogas and/or electric energy and the production of slabs and boards using the agave bagasse. It was also proposed to promote the formation of other companies, for example by taking the organic residues of greenhouses, seed and plants, to create a composting plant. With the proposed synergies, the waste generated by the agro-industrial sector could be reduced, as well as waste generated by companies with different activities, thus generating environmental and economic benefits to the municipality and the agro-industrial sector.

\section{INTRODUCCIÓN}

El sector agroindustrial comprende actividades de manufactura mediante las cuales se elaboran productos derivados del sector agrícola, principalmente para alimentación. Dentro de la industria agroalimentaria se encuentra la industria porcina. En México, en 2014, la producción de carne de porcino en canal ascendió a 1.29 millones de toneladas, y en 2015 se estimó en 1.32 millones de toneladas. En 2014, el sacrificio de ganado porcino para carne ascendió a 10.94 millones de cabezas, es decir, $8.7 \%$ menos que en 2013 (FIRA 2015).

El estado de Guanajuato es el sexto productor de carne de cerdo a nivel nacional y contribuye con el $8.4 \%$ de la producción, principalmente en el municipio de Irapuato y en menor proporción en Pénjamo; asimismo, en los municipios de Pénjamo y Celaya se encuentran establecidas algunas de las mayores empresas empacadoras de embutidos y procesamiento de carnes frías (FIRA 2015, Confederación de Porcicultores Mexicanos 2016).

En estas empresas, las instalaciones para sacrificio, que incluyen corrales de reposo, sacrificio, eliminación de la sangre, separación de piel o pelo, destripamiento y preparación para la venta, generan residuos de aproximadamente $18 \mathrm{t}$ por 1500 cerdos despiezados, compuestos esencialmente por vísceras, pelo y estiércol (Confederación de Porcicultores Mexicanos 2016). Estos residuos no reciben el tratamientos adecuado, con los consecuentes impactos ambientales como contaminación de agua, aire y suelos, pérdida y alteración de ecosistemas y biodiversidad, y contribución al cambio climático global (Gerber et al. 2013).

Según la Ley General para la Prevención y Gestión Integral de los Residuos (artículo 19), y en especial la Ley para la Gestión Integral de Residuos del Estado y los Municipios de Guanajuato (artículo 32), los residuos generados por actividades piscícolas, agrícolas, silvícolas, forestales, avícolas o ganaderas, incluyendo los residuos de los insumos utilizados en esas actividades, se clasifican como de manejo especial y por lo tanto están sujetos a planes de manejo que permitan su minimización, valorización y aprovechamiento (SEMARNAT 2003, IEEG 2005). Para la elaboración e implementación de planes de manejo de residuos de manejo especial se estableció la Norma Oficial Mexicana NOM-161-SEMARNAT-2011, que sin embargo no sugiere una metodología específica. $\mathrm{Su}$ única recomendación es que los planes sean factibles técnicamente, ambientalmente aceptables y económicamente sustentables (SEMARNAT 2013).

Ante la amplitud de opciones y, a la vez, ausencia de rutas claras para el desarrollo de planes de manejo efectivos en cuanto a minimización, valorización y aprovechamiento de los residuos, la ecología industrial, y dentro de ella la simbiosis industrial, surgen como alternativas innovadoras en el campo del aprovechamiento de los residuos, en este caso los del sector agroindustrial.

"La ecología industrial (EI) es un área interdisciplinaria, la cual intenta asimilar el funcionamiento de los ecosistemas industriales al de los naturales, con una interrelación entre industrias, el medio social y el natural, que tiende a cerrar el ciclo de materia y energía en una aproximación de los sistemas industriales hacia el desarrollo sostenible" (Cervantes 2013).

Esta relación entre industrias está en la línea de la economía circular, ya que trata de cerrar el ciclo de materia usando los residuos de una industria como materia prima de otras, con el objetivo de llegar a un nivel cero de residuos, tal como se presenta en los ecosistemas naturales (Lowe et al. 1997, Erkman 
2001, Isenmann 2003, Bossilkov et al. 2005, Cervantes et al. 2009, Cervantes 2013).

Un objetivo de la ecología industrial es la creación de sistemas de industrias interrelacionados que formen redes donde el intercambio no sea sólo material. La EI crea redes socioeconómicas que se vinculan mediante relaciones de cooperación, cliente-proveedor, de investigación, de pertenencia a asociaciones, de colaboración en proyectos comunes, etcétera. Otro objetivo de la EI es situar la actividad tecnológica como parte del ecosistema que la incluye, analizando la entrada de recursos y la salida de residuos, así como la manera en que la actividad humana afecta al ecosistema. Esta visión sistémica ha sido una de las grandes aportaciones de la ecología industrial (Erkman 2001, Cervantes 2013).

Dentro de la EI, la simbiosis industrial es el método o experiencia que relaciona entidades a través de un intercambio de materiales, energía, agua y residuos, creando redes materiales y sociales que promueven el cierre de ciclo de materia (Chertow 2000, 2007, Cervantes 2013).

Entre las sinergias que pueden establecerse dentro de la simbiosis industrial también pueden estar la utilización o implantación conjunta de un servicio o infraestructura. De igual modo, se llama simbiosis industrial al sistema de industrias interrelacionadas a través del método de simbiosis industrial, por lo que el mismo término define a la vez el método y la realidad que origina (Cervantes et al. 2009, Clift y Druckman 2015).

En este trabajo se aplican los principios de la ecología industrial a un grupo agroindustrial del municipio de Pénjamo, Guanajuato, México, para crear sinergias de materia y energía entre las empresas del grupo e industrias cercanas, a fin de que los residuos de unas puedan utilizarse como materia prima de otras en forma directa, y así optimizar la valorización de los residuos a partir de planes de manejo.

El grupo agroindustrial seleccionado, ubicado en el municipio de Pénjamo, produce alimentos y productos derivados de cerdo. Está asentado en un predio de 26 ha y comprende seis entidades de cuatro empresas distintas (Solís 2016):

1. Un rastro certificado con un sello TIF, lo cual indica que es un establecimiento Tipo Inspección Federal cuya actividad principal es la matanza y despiece de cerdos, con instalaciones de sacrificio de animales, frigoríficos e industrializadores de productos y subproductos cárnicos.

2. Una planta que procesa y comercializa la carne que proviene del rastro.
3. Una planta acondicionadora de vísceras rojas, que posteriormente se envían a empresas para hacer croquetas para perros.

4. Una planta de rendimiento que elabora suplementos alimenticios de consumo animal a partir de vísceras, huesos, sangre, pelo, pezuñas y también animales muertos en los corrales o en las canales, no apropiados para el consumo humano.

5. Una empresa dedicada al autotransporte de carga federal, que transporta carne fresca de cerdo y sus derivados.

6. Una empresa que proporciona servicios logísticos al grupo, cubriendo la gestión y planificación de las actividades de los departamentos de compras, producción, transporte, almacenaje, manutención y distribución, y conforma un sistema de enlace con la producción.

\section{MÉTODOS Y MATERIALES}

La metodología constó de cinco etapas. En la primera se determinaron los flujos de materia y energía de cada empresa del grupo agroindustrial por medio de visitas de campo y entrevistas con personal del grupo. Estos datos se representaron en diagramas de caja negra elaborados con el software Microsoft Office Visio 2003. Para la identificación de los flujos de materia y energía se usó el código de colores del Grupo de Investigación en Ecología Industrial (GIEI) de la Unidad Profesional Interdisciplinaria de Biotecnología (UPIBI), del Instituto Politécnico Nacional (IPN), el cual se muestra en el cuadro I (Lule y Cervantes 2010).

La segunda etapa consistió en detectar empresas ubicadas en un radio de $30 \mathrm{~km}$ tomando como centro el grupo, con las cuales pudieran establecerse sinergias de materia, energía, servicios o infraestructura. Para su reconocimiento se descargó el Directorio Estadístico Nacional de Unidades económicas (DENUE) (con extensión shape [shp]) del sitio web del Instituto Nacional de Estadística y Geografía (INEGI), para consulta y trabajo en un Sistema de Información Geográfica (QGIS). También se llevaron a cabo visitas de campo al municipio y localidades colindantes para reconocer las empresas localizadas alrededor del grupo, así como sus vías de comunicación.

En la tercera etapa, una vez localizadas e identificadas las empresas dentro del perímetro delimitado, se procedió por medio de visitas de campo, entrevistas e investigación, a conocer sus actividades, así como las materias primas, energía y agua que 
CUADRO I. CÓDIGO DE COLORES PARA DIAGRAMAS DE ECOLOGÍA INDUSTRIAL

\begin{tabular}{llrrrrr}
\hline Descripción & Flujo & Flujo residual & & \multicolumn{3}{c}{ Color con código RGB } \\
\cline { 4 - 7 } & & & $\mathrm{R}$ & $\mathrm{G}$ & $\mathrm{B}$ & Color \\
\hline Materias primas & & & 0 & 176 & 80 \\
Productos & & & & 204 & 0 & 255 \\
Energía & & & & 0 & 0 \\
Agua & & & & 0 & 112 & 192 \\
Residuos & & & & 127 & 96 & 0 \\
Sinergias propuestas & & & & 255 & 153 & 0 \\
\hline
\end{tabular}

R: rojo, G: verde, B: azul

Fuente: Lule y Cervantes 2010

utilizan en la elaboración de sus productos, además de la respectiva generación de residuos y productos. Asimismo, se investigó la existencia de servicios o infraestructuras compartidas y se determinó el potencial de habilidades adquiridas por las empresas para gestionar sus flujos residuales

En la cuarta etapa, con la información recabada anteriormente, se establecieron por un lado las sinergias existentes entre el grupo agroindustrial y las empresas localizadas en el perímetro de estudio y, por otro, los flujos residuales de materiales y energía de las empresas. Para establecer las sinergias existentes se tomó en cuenta una de las siguientes condiciones:

a. Sinergia de materia: se observó y registró si los residuos de las entidades son usados como materia prima de otra empresa y viceversa, o entre empresas aledañas.

b. Sinergia de flujo energético: Se investigó si existe algún flujo residual energético en el área y si es aprovechado por otra empresa.

c. Sinergia de infraestructura: Se estudió y registró si existe infraestructura común entre las entidades cercanas o entre las entidades vecinas.

d. Sinergia de servicios: Se reconoció si las entidades utilizan en conjunto un servicio. Posteriormente se realizó el diagrama de sinergias existentes, según el código de colores del cuadro I.

Finalmente, en la quinta etapa se procedió a estudiar y proponer nuevas sinergias para el grupo agroindustrial y su entorno, con el fin de cerrar el ciclo de materia y aprovechar la energía. Se investigaron experiencias regionales, nacionales e internacionales y aquéllas que las mismas empresas han tenido. Se identificaron dichas sinergias y se elaboró el diagrama de sinergias propuestas con el software Microsoft Visio 2003.

\section{RESULTADOS Y DISCUSIÓN}

El diagrama de caja negra del grupo agroindustrial se muestra en la figura 1, donde se observan residuos no aprovechados como pezuñas, párpados y pelo. Este último constituye una gran problemática dentro del grupo, ya que si bien puede utilizarse para hacer brochas de cerdas naturales, se encuentra mezclado con residuos de sangre, por lo que no puede valorizarse. Otros residuos que se encuentran en la misma situación son la sangre y vísceras verdes. Tampoco se aprovechan las llantas desechadas por la empresa de autotransporte del grupo ni las tarimas usadas, entre otras. Actualmente estos residuos se depositan en el tiradero del municipio o bien en fosas dentro de los terrenos del grupo, sin mayor tratamiento.

El diagrama de sinergias del grupo agroindustrial (Fig. 2) marca los flujos de materia y energía de las seis entidades; también indica los intercambios de productos y desechos entre entidades, por ejemplo los cerdos muertos o que no pasan la inspección son llevados a la planta de rendimiento del mismo grupo.

El grupo agroindustrial creó una red de simbiosis industrial con agrotecnología. Esto permite la valorización de subproductos y residuos cuyo monto asciende a $2954.47 \mathrm{t}$ tratados cada año, de las cuales el $97 \%$ se utiliza como materia prima para otras empresas (Sánchez 2015).

En el diagrama se advierten 15 sinergias, entre ellas la valorización de huesos, restos de carne no aptos para consumo humano y sangre, con los cuales se producen harinas de carne y sangre para la elaboración de alimentos pecuarios y/o de mascotas.

También se señala el aprovechamiento de las vísceras rojas, que son enviadas a la planta acondicionadora de vísceras para su posterior entrega a una empresa externa para la elaboración de alimento para mascotas. 


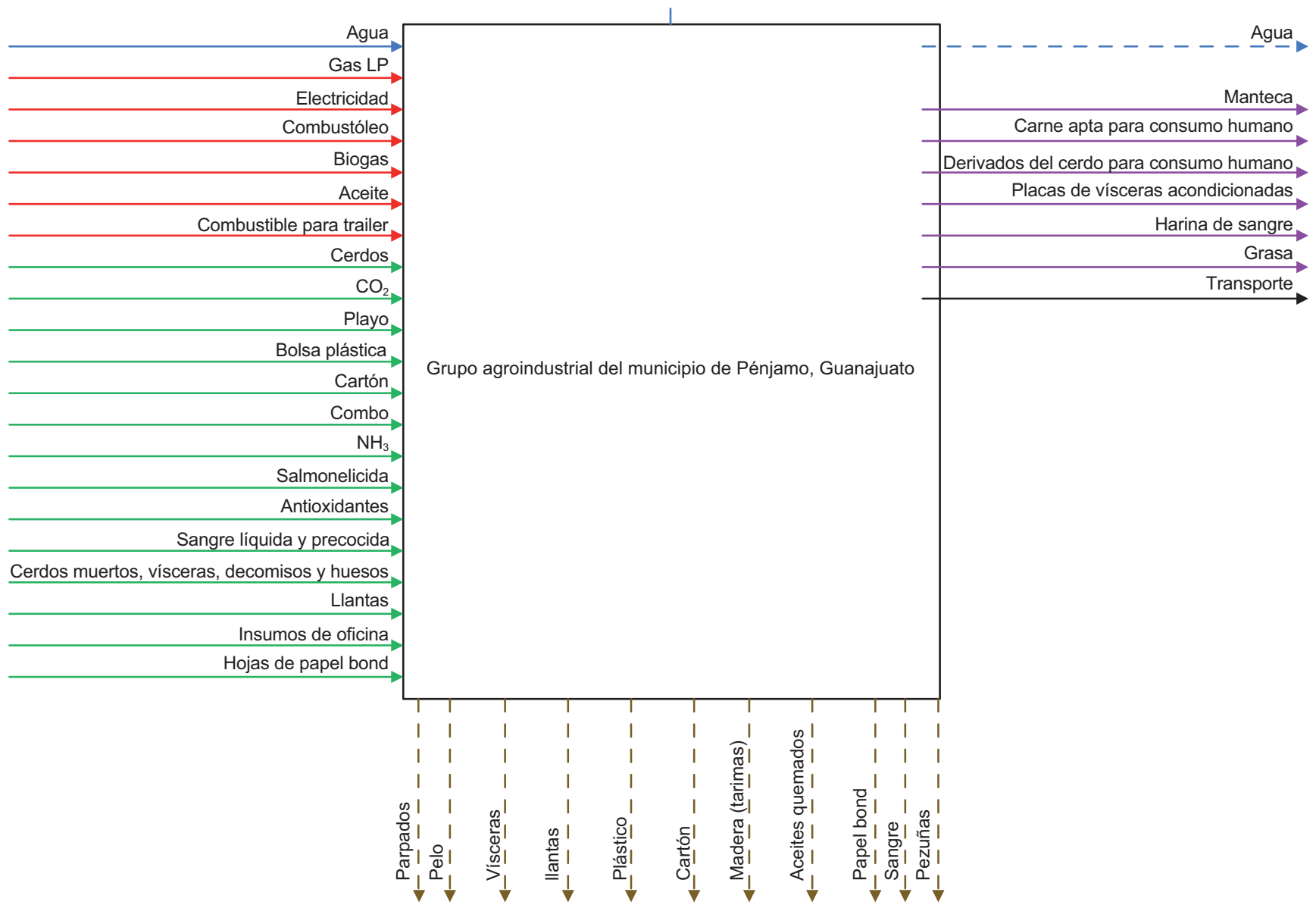

Fig. 1. Diagrama de caja negra del grupo agroindustrial (véase el código de colores en el Cuadro I)

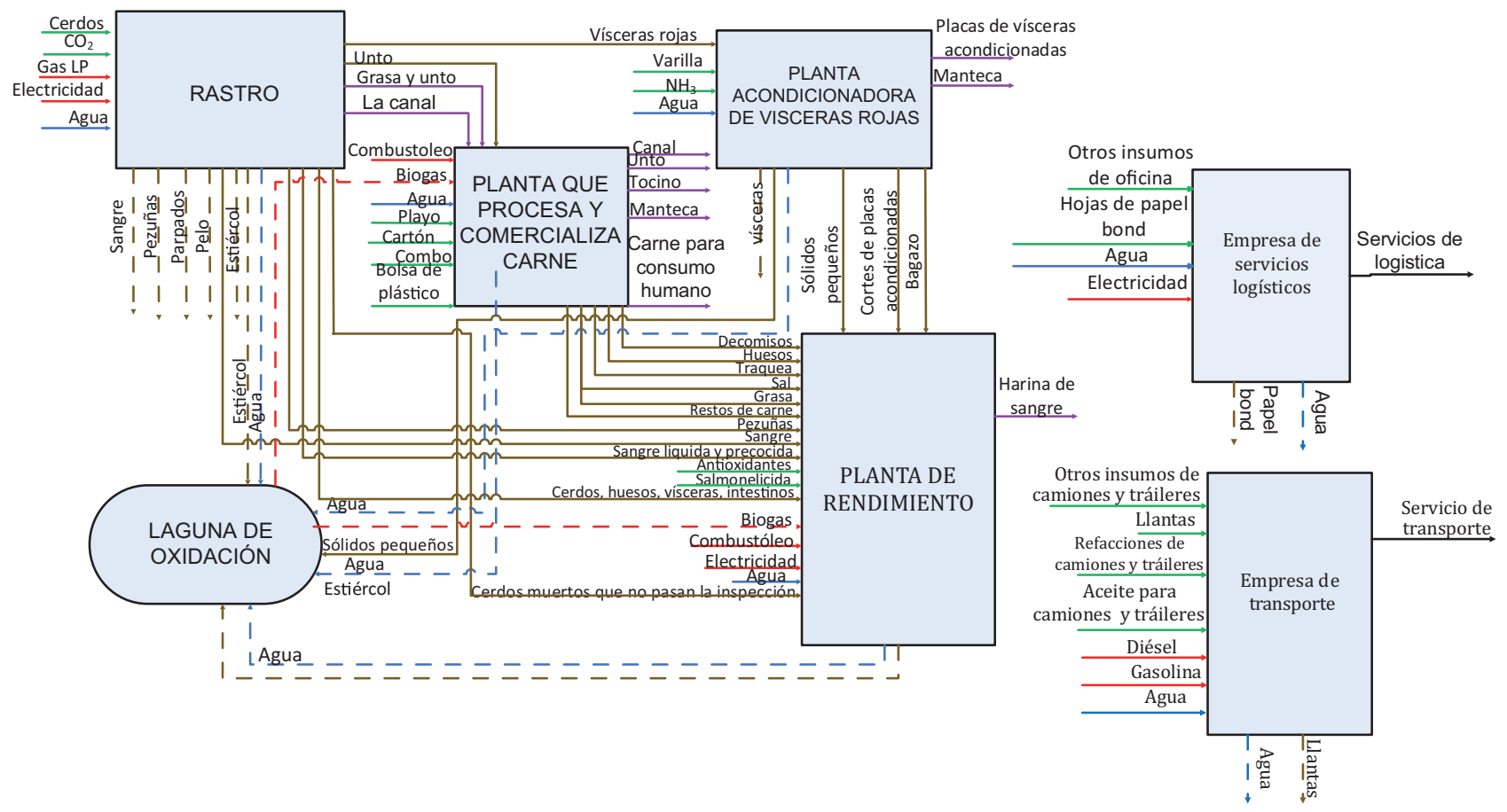

Fig. 2. Diagrama de sinergias dentro del grupo agroindustrial (véase el código de colores en el Cuadro I) 


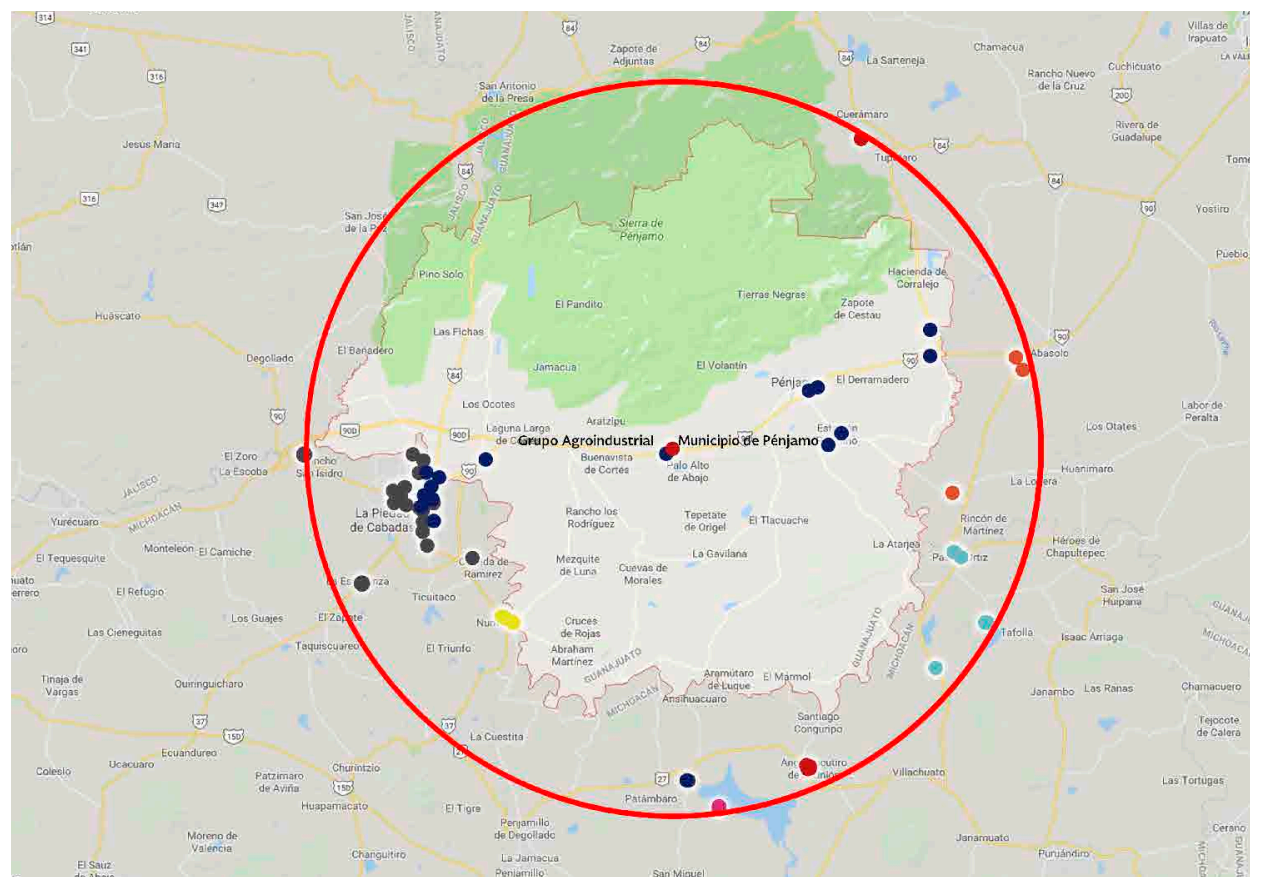

Fig. 3. Área de establecimiento de sinergias en torno al grupo agroindustrial de Pénjamo. (Fuente: INEGI 2015)

Asimismo se observa cómo el agua residual, el estiércol y los sólidos pequeños son enviados a una laguna de oxidación, de la cual se aprovecha el biogás obtenido en la planta dedicada al proceso y comercialización de carne, así como en la planta de rendimiento.

Sin embargo, el desarrollo tecnológico con que cuenta el grupo agroindustrial resulta insuficiente para la valorización del $100 \%$ de los flujos residuales. Si bien varios residuos se logran enviar a otras plantas y/o lagunas de oxidación dentro del grupo, un porcentaje no se aprovecha y se desecha en tiraderos municipales $\mathrm{y} / \mathrm{o}$ fosas.

Aunque el grupo agroindustrial cuenta con un área de carácter ambiental, ésta no desarrolla planes de manejo de los flujos residuales. Para estos residuos que no son aprovechados al interior del grupo se investigan opciones a través de la detección de sinergias entre el grupo y empresas aledañas, aplicando principios de la EI.

Tomando como centro el grupo agroindustrial se delimitó una circunferencia con un radio de $30 \mathrm{~km}$, la cual detecta aquellas empresas con las que el grupo podría establecer sinergias (Fig. 3). Se observa que el grupo se sitúa céntricamente en el municipio de Pénjamo, y que el perímetro de la zona de trabajo rebasa las fronteras del mismo. Por ello los intercambios de flujos podrían extenderse a otros municipios como Abasolo y Cuerámaro en Guanajuato; Penjamillo, Numarán, Angamacutiro y La Piedad en Michoacán, o Arandas en Jalisco.

Existen cerca de 110 empresas (incluyendo PYMES) dentro de la zona de estudio (INEGI 2015), de las cuales se incluyeron 18 en la muestra de estudio, por considerarse que tienen posibilidades de establecer sinergias. Las empresas seleccionadas (Cuadro II) se encuentran en tres estados, como se indicó antes, y tienen giros diversos: comercio de ganado, aves y animales en pie, destacando la producción de cerdos; empaquetadoras de carne de cerdo y su comercialización; elaboración de bebidas destiladas de agave; autotransportes con y sin refrigeración; invernaderos; comercio de combustibles, y fabricación de calzado y ladrillos.

La información concentrada sobre las materias primas, energía y agua que estas empresas usan en la elaboración de sus productos, así como su respectiva generación de residuos y productos, se presenta en el cuadro III. Para la información detallada véase Solís (2016).

En la figura 4 se muestran las sinergias existentes entre el grupo agroindustrial de Pénjamo y las empresas externas seleccionadas. Cabe mencionar que, como parte de estas sinergias, se incluyeron las 
CUADRO II. EMPRESAS SUSCEPTIBLES DE INTERCAMBIOS

\begin{tabular}{ll}
\hline Agroindustriales & Otras \\
\hline Frigorífico y Rastro de Santa Ana & $\begin{array}{l}\text { IPASA (metalmecánica) } \\
\text { Ladrilleras artesanales }\end{array}$ \\
\hline Granjas porcícolas de Santa Ana Pacueco, Gto. & MULTICO (elaboración de productos eléctricos) \\
\hline $\begin{array}{l}\text { Mantequera Cendejas } \\
\text { Planta de Carne en Estado Natural }\end{array}$ & Silver sport (maquila textil) \\
\hline $\begin{array}{l}\text { Grupo Agroindustrial de La Piedad (granja porcícola, planta } \\
\text { procesadora de carne, planta comercializadora de carnes y em- } \\
\text { butidos, planta de elaboración de alimento para animales, planta } \\
\begin{array}{l}\text { dedicada al acondicionamiento de semillas, planta de elaboración } \\
\text { de alimento para mascotas, invernadero de jitomates y pimientos, } \\
\text { planta de comercialización de fertilizantes) }\end{array}\end{array}$ \\
\hline \begin{tabular}{l} 
AER \\
\hline
\end{tabular}
\end{tabular}

CUADRO III. RESUMEN DE MATERIAS PRIMAS, CONSUMO DE ENERGÍA, AGUA Y RESIDUOS GENERADOS EN LAS EMPRESAS SELECCIONADAS

\begin{tabular}{|c|c|c|}
\hline Tipo de empresa & Insumos & Flujos residuales \\
\hline \multirow{4}{*}{ Granjas porcícolas } & Cerdos en pie & \multirow{2}{*}{ Purines } \\
\hline & Alimento pecuario & \\
\hline & Agua & \multirow[b]{2}{*}{ Agua residual } \\
\hline & Energía eléctrica & \\
\hline \multirow{6}{*}{ Invernaderos } & Semillas & Mermas de plantas \\
\hline & $\underline{\text { Plántulas }}$ & Envases fitosanitarios \\
\hline & Sustratos & Poliestireno expandido (unicel) \\
\hline & Agua & Agua residual con alto contenido en sodio \\
\hline & Energía eléctrica & Mermas de semillas \\
\hline & Gas natural & Mermas de jitomate \\
\hline \multirow{5}{*}{ Frigoríficos } & Cerdos & Vísceras \\
\hline & Insumos de sanidad & \multirow{2}{*}{ Pelo } \\
\hline & Agua & \\
\hline & Energía eléctrica & \multirow{2}{*}{ Agua residual } \\
\hline & Combustóleo & \\
\hline \multirow{5}{*}{ Procesadoras de carne } & Canales & Huesos \\
\hline & \multirow[t]{2}{*}{ Bolsas plásticas } & Vísceras \\
\hline & & Sangre \\
\hline & \multirow[t]{2}{*}{ Tarimas } & Plástico \\
\hline & & Agua residual \\
\hline \multirow{4}{*}{ Tequilera } & Agave & Residuos de agave \\
\hline & Energía eléctrica & Vinazas \\
\hline & Envases & Tarimas \\
\hline & Gas LP & Agua residual con nitrógeno \\
\hline
\end{tabular}




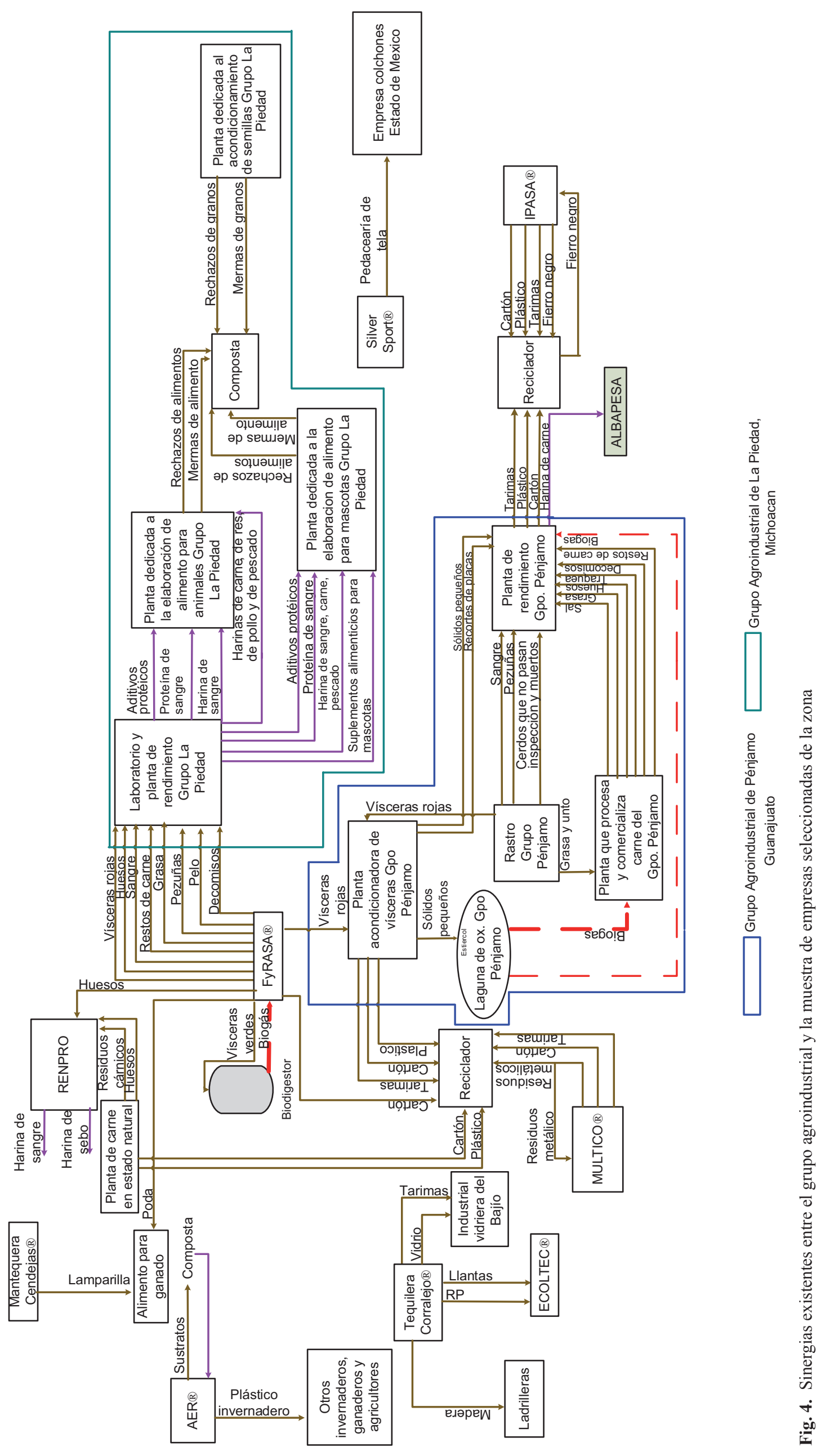


lagunas de oxidación y los biodigestores. Aunque estas instalaciones no son entidades productivas, sirven para el aprovechamiento de flujos residuales de agua y materia en la generación de biogás y/o electricidad.

La figura 4 muestra la existencia de dos grupos agroindustriales que manejan la simbiosis industrial: el de Pénjamo (Guanajuato) y el de La Piedad (Michoacán). Ambos envían subproductos y residuos a otras entidades para la elaboración de productos. Se destaca que la mayoría de las sinergias establecidas, ya sea dentro de los grupos o con empresas externas, están esencialmente relacionadas con la industria porcina y con la valorización de los residuos de cerdo después de su acondicionamiento para consumo humano. Dentro del grupo de La Piedad resaltan seis sinergias que favorecen el aprovechamiento de rechazos de alimentos y de grano para la elaboración de composta. Este grupo también recibe nueve subproductos y residuos generados por la empresa Fyrasa, entre los cuales se encuentran pelo, pezuñas, sangre y vísceras rojas, que son enviados al laboratorio y planta de rendimiento. Con estos productos se fabrican aminoácidos para saborizantes artificiales así como aditivos proteicos, que posteriormente son usados para la elaboración de alimentos pecuarios y de mascotas en una planta del mismo grupo.

Asimismo, es visible el uso de la técnica de compostaje, tanto para las plantas de granos y/o alimentos pecuarios o de mascotas, como para invernaderos de jitomates y la industria tequilera. También se observa el uso de biodigestores para la obtención de biogás, y posterior uso en las plantas que lo generan. Otras sinergias reflejadas que no se relacionan con la industria porcina son el reúso de tarimas, plástico y cartón como complementos en las empresas para embalaje de productos y/o materias primas.

Se detectaron 39 tipos de flujos residuales generados por el grupo y las empresas que no son aprovechados. En el cuadro IV se mencionan los principales, entre los que se encuentran residuos como pelo, párpados y pezuñas de cerdo, bagazo de agave, llantas y vísceras verdes, entre otros. Para la información detallada véase Solís (2016). Es importante señalar que un tipo de residuo puede ser generado por más de una empresa en el perímetro de estudio. Como se puede observar, la mayoría de los residuos se depositan en tiraderos o fosas. Para establecer propuestas de nuevas sinergias y posibles intercambios entre todas las empresas del perímetro de estudio para la valorización de residuos, se tomaron en cuenta los siguientes parámetros: a. Empresas viables para utilizar los residuos como materia prima, ubicadas dentro del perímetro de trabajo.

b. Empresas fuera de la zona con la posibilidad incluso de proponer nuevas sinergias, las cuales podrían extender la red de ecología industrial a otras regiones.

c. Proyectos generados por las empresas para la gestión de sus residuos.

d. Experiencias regionales, nacionales y/o internacionales de aprovechamiento de residuos, poniendo atención en las que actualmente son utilizadas por empresas dedicadas al reciclaje de materiales.

e. Pruebas piloto de aprovechamiento de residuos.

Las propuestas de nuevas sinergias y posibles intercambios entre empresas se pueden consultar en el cuadro IV y la figura 5.

Se construyeron alternativas de aprovechamiento para 25 tipos de flujos residuales, y en algunos casos, como mermas de granos, alimentos pecuarios y/o mascotas, se pudo crear más de una sinergia. Se establecieron 45 sinergias entre 23 empresas de los estados considerados, de ramos parecidos (principalmente enfocados al sector agroindustrial) o muy diferentes (como el coprocesamiento de residuos).

Se propone que el pelo de cerdo, generado por el grupo de Pénjamo, sea enviado al grupo de La Piedad para aprovecharse en la misma forma que en la empresa Fyrasa, es decir, en la elaboración de saborizantes artificiales y aditivos proteicos. Asimismo puede utilizarse para la producción de cosméticos y/o en la industria farmacéutica. Los parpados y pezuñas pueden ser utilizados por alguna empresa grenetinera ya existente o que se instale dentro del área.

Las fibras del bagazo de agave generado por la industria tequilera después de la destilación pueden usarse mezcladas con polímeros para la obtención de un material con aplicaciones similares a la madera. Otra aplicación es la fabricación de láminas acanaladas, que permitirían evitar el uso de materiales como el asbesto.

$\mathrm{El} \mathrm{CO}_{2}$ generado por las tequileras puede utilizarse en los invernaderos, los cuales emplean este gas como sustrato para el crecimiento de las plantas. Actualmente lo obtienen del proceso de calentamiento de agua y/o lo compran a empresas químicas, lo cual eleva el precio del producto debido al transporte del producto. También puede ser aprovechado por el rastro del grupo de Pénjamo, el cual maneja una cámara con este gas para el proceso de matanza del cerdo.

Los residuos de aceites de taller quemados, de envases de fertilizantes y medicamentos, así como 
CUADRO IV. FLUJOS RESIDUALES NO APROVECHADOS Y SU MANEJO ACTUAL

\begin{tabular}{|c|c|}
\hline Residuo & Manejo actual \\
\hline Aceite de fusel & Almacenado en la planta \\
\hline Aceite de taller & Enviado al tiradero municipal (de Pénjamo o La Piedad) \\
\hline \multirow{2}{*}{ Agua de retrolavado (c/sodio) } & Usada para riego de caminos \\
\hline & Vertida en drenaje \\
\hline Bagazo & Enviado al tiradero municipal (de Pénjamo o La Piedad) \\
\hline Bolsas con grasa & Enviadas al tiradero municipal (de Pénjamo o La Piedad) \\
\hline Cerdos muertos & Depositados en fosas \\
\hline $\mathrm{CO}_{2}$ & Emitido a la atmósfera \\
\hline Descargas de granjas (purines) & Enviadas a lagunas de oxidación \\
\hline \multirow{2}{*}{$\begin{array}{l}\text { Envases de fertilizantes y plagui- } \\
\text { cidas }\end{array}$} & Enviados al tiradero municipal (de Pénjamo o La Piedad) \\
\hline & Depositados en el invernadero \\
\hline Envases de medicamentos & Enviados a tiradero municipal (de Pénjamo o La Piedad) \\
\hline \multirow{2}{*}{ Estiércol } & $\underline{\text { Depositado en fosas }}$ \\
\hline & Enviado a laguna de oxidación del grupo agroindustrial del municipio de Pénjamo \\
\hline \multirow{2}{*}{ Hojas bond } & Enviadas al tiradero municipal (de Pénjamo o La Piedad) \\
\hline & Enviadas a reciclaje \\
\hline Ladrillos echados a perder & Depositados en el sitio \\
\hline Lamparilla & Enviada a granjas para alimento para ganado \\
\hline Lana de roca & Depositada en sitio \\
\hline Ligas & Enviadas al tiradero municipal (de Pénjamo o La Piedad) \\
\hline \multirow{3}{*}{ Llantas } & Enviadas al tiradero municipal (de Pénjamo o La Piedad) \\
\hline & Almacenadas en el sitio donde se generaron \\
\hline & Vendidas a refaccionarias \\
\hline Madera & Regalada a personas que tienen estufas de leña \\
\hline \multirow{2}{*}{$\begin{array}{l}\text { Mermas de alimentos pecuarios } \\
\text { y de mascotas }\end{array}$} & Enviadas al tiradero municipal (de Pénjamo o La Piedad) \\
\hline & Compostaje \\
\hline Mermas de granos & Compostaje \\
\hline Mermas y restos de plántulas. & Enviadas a tiradero municipal (de Pénjamo o La Piedad) \\
\hline Moldes de cerámica & Enviados a tiradero municipal (de Pénjamo o La Piedad) \\
\hline Overoles & Enviados a tiradero municipal (de Pénjamo o La Piedad) \\
\hline \multirow{2}{*}{ Párpados } & Depositados en fosas \\
\hline & Enviados al tiradero municipal (de Pénjamo y/o La Piedad) \\
\hline \multirow{3}{*}{ Pelo } & Depositado en fosas \\
\hline & Enviado al tiradero municipal (de Pénjamo o La Piedad) \\
\hline & $\begin{array}{l}\text { Enviado al laboratorio de investigación y planta de rendimiento del grupo agroindustrial de La } \\
\text { Piedad }\end{array}$ \\
\hline \multirow{2}{*}{ Pezuñas } & Depositadas en fosas \\
\hline & Enviadas al tiradero municipal (de Pénjamo o La Piedad) \\
\hline
\end{tabular}


CUADRO IV. FLUJOS RESIDUALES NO APROVECHADOS Y SU MANEJO ACTUAL

\begin{tabular}{|c|c|}
\hline Residuo & Manejo actual \\
\hline Pinceles & Enviados al tiradero municipal (de Pénjamo o La Piedad) \\
\hline \multirow{2}{*}{ Plástico de invernadero } & Depositado en el invernadero \\
\hline & Enviado a otros invernaderos \\
\hline \multirow{2}{*}{ Poda } & $\underline{\text { Regalada a personas que mantienen animales de ganado }}$ \\
\hline & Enviada al tiradero municipal (de Pénjamo o La Piedad) \\
\hline \multirow{2}{*}{ Purines (excretas y orina) } & Vertidos en drenaje \\
\hline & Enviados a lagunas de oxidación \\
\hline \multirow{2}{*}{ Rafia } & Enviada al tiradero municipal (de Pénjamo o La Piedad) \\
\hline & Depositada en el invernadero \\
\hline \multirow{2}{*}{$\begin{array}{l}\text { Rechazos de alimentos } \\
\text { pecuarios y de mascotas }\end{array}$} & Enviados al tiradero municipal (de Pénjamo o La Piedad) \\
\hline & Reenviados al proceso o compostaje \\
\hline Rechazos de granos & Reenviados al proceso o compostaje \\
\hline \multirow{3}{*}{ Residuos cárnicos } & Depositados en fosas \\
\hline & Enviados al tiradero municipal (de Pénjamo o La Piedad) \\
\hline & Enviados a la planta de rendimiento RENPRO \\
\hline Residuos metálicos (refacciones) & Sin información \\
\hline Suero de lecha de cabra & Vertido en drenaje \\
\hline \multirow{3}{*}{ Sustratos, sustrato de coco } & Enviados al tiradero municipal (de Pénjamo o La Piedad) \\
\hline & Depositados en el invernadero \\
\hline & Compostaje \\
\hline \multirow{2}{*}{ Unicel } & Enviado al tiradero municipal (de Pénjamo o La Piedad) \\
\hline & Depositado en sitio \\
\hline \multirow{4}{*}{ Víscera verde } & Enviada a biodigestor \\
\hline & Enviada a la planta de rendimiento del grupo agroindustrial del municipio de Pénjamo \\
\hline & Enviada al tiradero municipal (de Pénjamo o La Piedad) \\
\hline & Depositado en fosas \\
\hline
\end{tabular}

las llantas, pueden tener como fin el reciclaje térmico proporcionado por la empresa Ecoltec, que posee una planta de recolección de residuos en la zona. Estos residuos pueden acopiarse en el relleno sanitario del municipio de Pénjamo y enviarse a la misma planta de recolección.

Los plásticos, playo, rafia y unicel provenientes del embalaje de materias primas y/o productos, $\mathrm{y}$ las hojas bond pueden ser valorizados por empresas recicladoras. En la zona se encontraron diferentes PyMES que pueden ser enlace entre las entidades de trabajo y las grandes recicladoras.

La sangre puede aprovecharse en las plantas de rendimiento de los dos grupos agroindustriales para la fabricación de alimentos destinados a animales domésticos y de granjas.
Un flujo residual de todas las empresas son las tarimas de madera, que pueden enviarse a acopiadores para que a su vez sean remitidas a plantas recicladoras. Las tarimas que ya no puedan recuperarse pueden servir como insumo para las ladrilleras artesanales, evitando así la quema de llantas y aceites contaminados en la zona.

Otro grupo importante de residuos (debido a su volumen y cantidad) generados por más de una empresa, son los de carácter orgánico. Se encontraron 21 tipos diferentes derivados propiamente de las actividades agroindustriales, entre los cuales se encuentran cerdos muertos, estiércol, lana de roca, mermas de plántulas, poda, sustrato de coco y vísceras verdes. Estos residuos pueden enviarse a la empresa gestora de residuos no peligrosos del grupo agroindustrial 


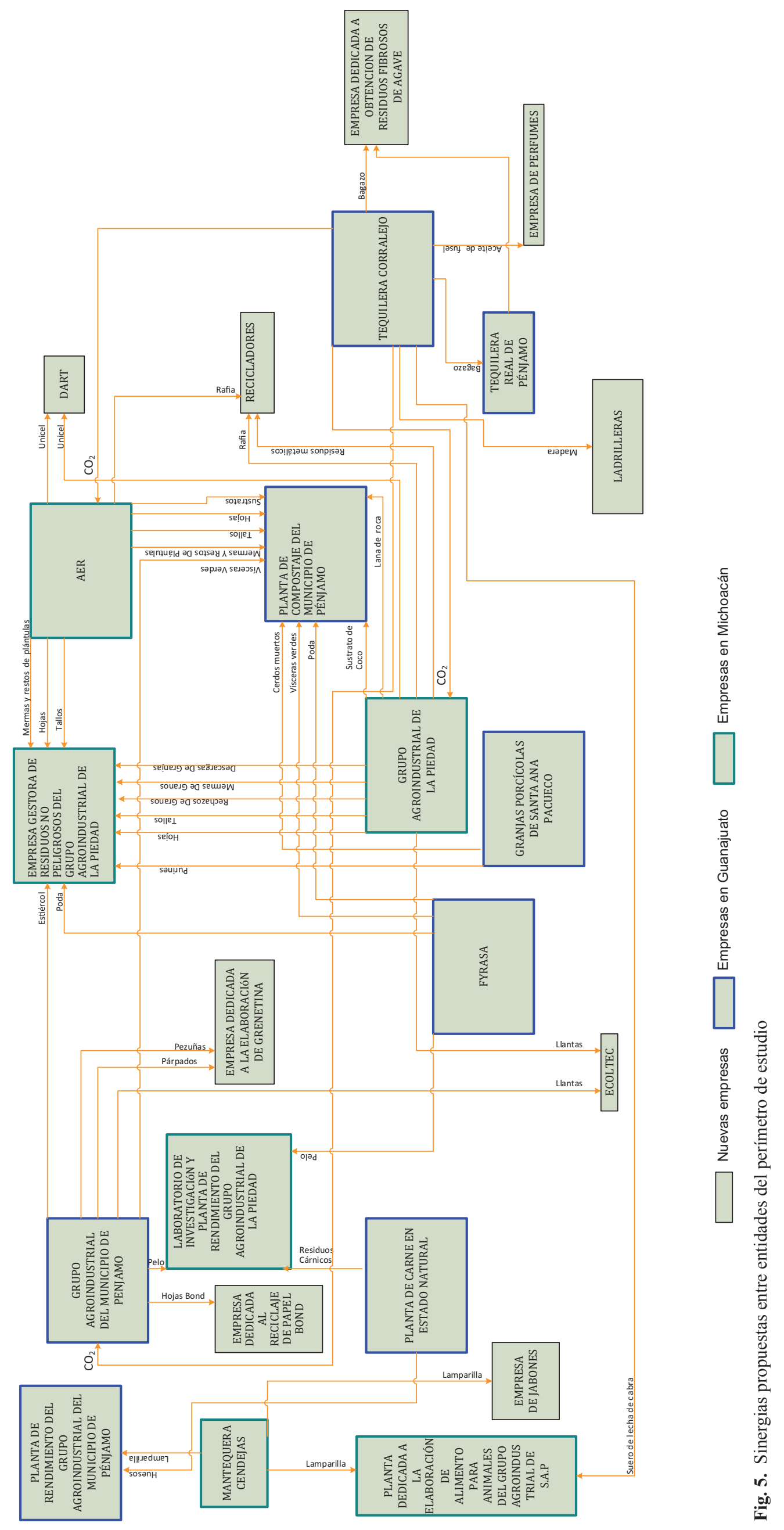


de La Piedad, que planea construir un biodigestor para valorizar los purines generados por las granjas porcícolas del grupo, así como los restos de las plántulas de su propio invernadero. En este tenor, podría también coadyuvar con la gestión de los residuos de otras granjas porcícolas, así como de los residuos orgánicos generados en la zona. Este biodigestor se propone como una nueva empresa.

Dentro de las sinergias existentes se señala que una planta de composta del grupo de la Piedad podría ampliarse y recibir más residuos orgánicos generados por las empresas de la zona por el municipio, lo cual podría gestionarse con inversión público-privada; sin embargo, los subproductos resultantes no son utilizados en el área, aun cuando se cuenta con invernaderos dedicados a la cosecha de especies vegetales. Por ello el método más apto para la valorización de estos residuos sería la construcción de un biodigestor para la obtención de energía y/o gas resulta ser.

Finalmente, otra manera de gestionar los residuos sería mediante de una bolsa de subproductos, que es un sistema de intercambio de residuos entre empresas, usualmente a través de una comunicación virtual en una plataforma en línea. Las empresas anuncian sus residuos y otras empresas las compran. La bolsa de subproductos se convierte así en un medio por cual las empresas de distintos sectores pueden difundir e intercambiar subproductos o residuos que otras empresas pueden utilizar como materia prima en la elaboración de sus propios productos (Hernández y Fernández 2013).

En la elaboración de un plan de manejo de residuos especiales, la etapa de organización y diseño de las formas de aprovechamiento de los residuos es la más importante, ya que ahí se decide todo el flujo de residuos; también es la más compleja, porque las soluciones conocidas de aprovechamiento no siempre son pertinentes o suelen ser costosas. Los resultados obtenidos en esta investigación mostraron que las herramientas de la ecología industrial y la simbiosis industrial, a través de la búsqueda sistemática y metódica de opciones de intercambios entre empresas existentes o con nuevas estructuras, abren un panorama nuevo con alternativas viables y más satisfactorias para las partes.

\section{CONCLUSIONES}

Se pudo desarrollar una propuesta de valorización de los residuos no aprovechados de un grupo agroindustrial del municipio de Pénjamo, Guanajuato, México, y de otras entidades colindantes con dicho estado, usando los flujos de residuos de unas empresas como materias primas para otras. De esta manera se mostró una forma diferente de establecer planes de manejo de residuos especiales, aplicando principios de ecología industrial.

Para ello se identificaron los flujos de materia y energía, los intercambios de productos y desechos entre las seis entidades que componen el grupo agroindustrial, así como los flujos residuales no aprovechados por éste, como pelo, párpados y pezuñas de cerdo, sangre, vísceras verdes, llantas, y tarimas.

Se localizaron alrededor del grupo agroindustrial del municipio de Pénjamo, 18 empresas y otro grupo agroindustrial, susceptibles de interrelacionarse con el grupo antes mencionado para llevar a cabo un intercambio de materia y/o energía. Tanto de estas 18 empresas como del segundo grupo agroindustrial se determinaron flujos de materia y energía de manera cualitativa, así como las sinergias establecidas a partir del intercambio de materia y energía, o el uso de conjunto de servicios y/o infraestructura.

También se encontraron 39 tipos de flujos residuales generados por el clúster agroindustrial que no eran aprovechados y que, en su mayoría, eran depositados en tiraderos o fosas. Con la aplicación de los principios de la ecología industrial se obtuvieron formas de valorización para 25 de estos flujos, creándose 45 sinergias con 23 empresas de ramos parecidos (dedicadas principalmente al sector agroindustrial) o muy diferentes, ubicadas en la zona que abarca a municipios de Guanajuato, Michoacán y Jalisco.

Se propusieron proyectos para la obtención de beneficios comunes entre empresas, como el caso del $\mathrm{CO}_{2}$ emitido por una empresa tequilera que puede ser aprovechado por los invernaderos y por el rastro del grupo agroindustrial de Pénjamo, o el pelo de cerdo que puede ser utilizado para la fabricación de saborizantes artificiales y aminoácidos. La planta de composta existente podría ampliarse para recibir los residuos orgánicos de invernaderos, mermas de semillas y alimentos, y podría contribuir a la gestión de residuos no sólo de la agroindustria, sino también del municipio.

Finalmente, se plantearon proyectos nuevos como plantas dedicadas a la producción de grenetina a partir de pezuñas y párpados, o la elaboración de láminas y tableros utilizando el bagazo de agave. También se propusieron estructuras nuevas como biodigestores en los que se utilizarían los residuos y purines de granjas porcícolas como sustrato para la obtención de biogás y/o energía eléctrica. 


\section{REFERENCIAS}

Bossilkov A., van Berkel R. y Corder G. (2005). Regional synergies for sustainable resource processing: A status report. Project 3A1: Enabling tools and technologies for capturing regional synergies. Centre for Sustainable Resource Processing, Queensland, Australia [en línea]. https://espace.library.uq.edu.au/view/UQ:346084

Cervantes G., Sosa G.R., Rodríguez H.G. y Robles M.F. (2009). Ecología industrial y desarrollo sustentable. Ingeniería Revista Académica de la Facultad de Ingeniería de la Universidad Autónoma de Yucatán 13 (1), 63-70.

Cervantes G. (2013). El concepto de ecología industrial. En: La ecología industrial en México (Carrillo G., Ed.). Universidad Autónoma Metropolitana-Unidad Xochimilco, Ciudad de México, 47-68.

Chertow M.R. (2000). Industrial symbiosis: Literature and taxonomy. Annu. Rev. Energ. Env. 25(1), 313-337. DOI: 10.1146/annurev.energy.25.1.313

Chertow M.R. (2007). "Uncovering" industrial symbiosis. J. Ind. Ecol. 11(1), 11-30. DOI: 10.1162/jiec.2007.1110

Clift R. y Druckman A. (2015). Taking stock of industrial ecology. Springer, 362 pp. DOI: 10.1007/978-3-31920571-7

Confederación de Porcicultores Mexicanos (2016). Principales productores de carne [en línea]. http://www. porcimex.org/estadisticas.htm 06/02/2009

Erkman S. (2001). Industrial ecology: A new perspective on the future of the industrial system. Swiss Med. Wkly. 131(37-38), 531-538. DOI: 10.4414/ smw.2001.09845

FIRA (2015). Panorama agroalimenticio. Carne de porcino 2015. Dirección de Investigación y Evaluación Económica y Sectorial, Fideicomisos Instituidos en Relación con la Agricultura [en línea].https://www. gob.mx/cms/uploads/attachment/file/61951/Panorama_Agroalimentario_Carne_Porcino_2015.pdf

Gerber P.J., Steinfeld H., Henderson B., Mottet A., Opio C., Dijkman J., Falcucci A. y Tempio, G. (2013). Tackling climate change through livestock - A global assessment of emissions and mitigation opportunities. Food and Agriculture Organization of the United Nations, Roma [en línea]. http://www.fao.org/docrep/018/ i3437e/i3437e00.htm 08/06/2016

Hernández M.R. y Fernández G.S. (2013). Bolsa de subproductos: una estrategia de ecología industrial. En: La ecología industrial en México (Carrillo G., Ed.). Universidad Autónoma Metropolitana-Unidad Xochimilco, Ciudad de México, 289-311.
IEEG (2005). Ley para la gestión integral de residuos del estado y los municipios de Guanajuato. Instituto de Ecología del Estado de Guanajuato. Periódico Oficial del Estado de Guanajuato 74, segunda parte, 10 de mayo.

INEGI (2015). Directorio estadístico nacional de unidades económicas. Instituto Nacional de Estadística y Geografía [en línea]. en: http://www.beta.inegi.org.mx/app/ mapa/denue/default.aspx 20/02 2015

Isenmann R. (2003). Industrial ecology: Shedding more light on its perspective of understanding nature as model. Sustain. Dev. 11(3), 143-158.

DOI: $10.1002 / \mathrm{sd} .213$

Lowe E.A., Warren J.L. y Moran S.R. (1997). Discovering industrial ecology: An executive briefing and sourcebook. Battelle Press, Columbus, OH, 191 pp.

Lule C.D. y Cervantes T.M.G. (2010). Diagramas de flujo de sistemas industriales, una herramienta para la ecología industrial. El caso del corredor industrial de Altamira. Memorias del $5^{\circ}$ Congreso Internacional de Sistemas de Innovación para la competitividad 2010, Tecnologías Convergentes para la Competitividad, Celaya, México [en línea]. http://docplayer.es/4416083-Diagramas-deflujo-de-sistemas-industriales-una-herramienta-parala-ecologia-industrial-el-caso-del-corredor-industrialde-altamira.html 01/06/2015

Sánchez L.P. (2015). Sustainable agro-technological cluster in Mexico: The case of Pénjamo as a model for agroindustrial symbiosis systems. Cartel presentado en la ISIE Conference, Guildford, Reino Unido.

SEMARNAT (2003). Ley General para la Prevención y Gestión Integral de los Residuos Sólidos. Secretaría de Medio Ambiente y Recursos Naturales. Diario Oficial de la Federación, México, 8 de octubre (actualizada el 7 de junio de 2013).

SEMARNAT (2013). NOM-161-SEMARNAT-2011. Que establece los criterios para clasificar a los Residuos de Manejo Especial y determinar cuáles están sujetos a Plan de Manejo; el listado de los mismos, el procedimiento para la inclusión o exclusión a dicho listado; así como los elementos y procedimientos para la formulación de los planes de manejo. Secretaría de Medio Ambiente y Recursos Naturales. Diario Oficial de la Federación, México, 1 de febrero de 2013.

Solís J.E. (2016). Propuesta de ecología industrial para un grupo agroindustrial en el municipio de Pénjamo (Guanajuato). Proyecto terminal de la Licenciatura en Ingeniería Ambiental. Universidad Autónoma Metropolitana-Unidad Azcapotzalco, Ciudad de México. 